\title{
Improvement of Current Limiting and Recovery Characteristics of Flux-Lock Type SFCL with Series Connection of Two Coils Using Its Third Coil
}

\author{
Seok-Cheol Ko*, Young-Pil Kim** and Sung-Hun Lim ${ }^{\dagger}$
}

\begin{abstract}
In this work, the current limiting and recovery characteristics of a flux-lock type superconducting fault current limiter (SFCL) with series connection of two coils were effectively improved by adding a third winding into the conventional flux-lock type SFCL with series connection of two coils. To confirm the contribution of the third winding to the current limiting and recovery characteristics of this type of the SFCL, short-circuit testing was carried out with consideration of the third winding, and the effect of the third winding on the current limiting and recovery characteristics was examined by comparative analysis of the amplitude of the limited fault current and the power burden of the high-TC superconducting (HTSC) element comprising the SFCL. Through the analysis of both the limiting impedance and the operational current as the main design parameter of the SFCL, the improved current limiting and recovery characteristics of the flux-lock type SFCL using the third winding could be verified.
\end{abstract}

Keywords: Superconducting fault current limiter (SFCL), Fault current limiting, HTSC element, Recovery characteristics

\section{Introduction}

The increase of large-scale generation facilities with the drastic increase of the power demand has led to an increase in fault current due to short-circuit accidents along with decreased power grid impedance and increased complexity of the power grid. There is a rising interest in the development of new conceptual protection devices which can solve the problems related to these issues, such as, the grid instability due to bus separation, the influence on the grid during steady-state operation, the need to replace circuit breakers (including the associated cost increase) due to the increase of short-circuit current, and so forth [1-3]. There have been efforts to develop a fault current limiting device that can limit steadily increasing shortcircuit current, including researches on a high-temperature superconducting fault current limiter (SFCL), which may provide more effective control and limiting of fault current [4-6]. It has been reported that among SFCLs that use magnetic coupling operated by a single high-temperature superconducting (HTSC) element using a single iron core as a medium, flux-lock SFCLs (in which two coils are connected in parallel or in series) have adjustable

$\dagger$ Corresponding Author: Department of Electrical Engineering, Soongsil University, Seoul, Korea. (superlsh73@ssu.ac.kr)

* Cooperation Foundation, Kongju National University, Chungnam, Korea. (suntrac@kongju.ac.kr)

** Division of Electrical, Electronic and Control Engineering, Kongju National University, Chungnam, Korea. (suntrac@kongju.ac.kr)

Received: April 7, 2016; Accepted: September 29, 2016 current limiting capability, and the resistance of the HTSC element can also be adjusted higher or lower as well than that the resistive type SFCL [7-9]. Recently, a flux-lock SFCL model with the peak current limiting function using two separated SC modules has been introduced and its effective current limiting characteristics have been described [10-12].

With another SFCL model that uses magnetic coupling, we propose an SFCL structure that, with a single iron core as a medium, connects two coils in a series, adding an additional third winding to connect in parallel with the secondary coil, and this connects in series with the HTSC element. This structure is proposed to further improve the fault current limiting and recovery characteristics compared to the conventional SFCL in which two coils are connected in series. This paper compares and analyzes the fault current limiting and recovery characteristics with and without the third winding. In addition, the resistance characteristics of the HTSC element are analyzed and compared without the third coil in relation to the winding direction between the secondary and third coils. Through a short-circuit simulation experiment before and after fault occurrence, the fault current limiting and recovery characteristics were analyzed in relation to the winding direction between the third and secondary coils after the primary and secondary coils were set in an additive polarity winding direction. The analysis results, verified that these two characteristics were improved in comparison to the conventional SFCL where two coils are connected in a series. 


\section{Structure and Operation Principle}

\subsection{Structure and principle}

The typical SFCL structure largely consists of two magnetically coupled coils and HTSC elements. The proposed structure, as shown in Fig. 1, has a structure in which a single iron core is used as a medium to connect in series between the primary and secondary coil with additive winding. A third coil is added for parallel connection with the secondary coil, and the HTSC element is connected in a series with the third coil. Under normal conditions, two magnetic fluxes from two the coils are canceled out; thus, zero voltages are induced in the two coils when a third coil is included. If the current of the HTSC element increases and exceeds its critical current due to a fault accident, resistance of the HTSC element occurs, which causes a voltage across each of the coils to be induced. Therefore, the limiting impedance of this SFCL appears, and the fault current can be limited.

\subsection{Equivalent circuit}

Fig. 2 shows the electrical equivalent circuit of the SFCL with a series connection between two coils and an additional third coil. $L_{1}, L_{2}$, and $L_{3}$ denote the inductances of the three coils and $R_{s c}$ shows the resistance of the HTSC element. To simplify the analysis of the equivalent circuits, the mutual inductance among the coils is omitted. Under the assumption that the leakage impedance, the resistance of each winding, and iron loss can be ignored, the limiting impedance $Z_{F C L}$ of the SFCL can be expresses as follows:

$$
\begin{aligned}
V_{S F C L}= & j \omega\left(\sqrt{L_{1}}+\sqrt{L_{2}}\right)^{2} I_{1} \\
& \pm j \omega\left(\sqrt{L_{1}}+\sqrt{L_{2}}\right)\left(\sqrt{L_{3}} \mp \sqrt{L_{2}}\right) \times \\
& \left\{\frac{\left(\sqrt{L_{1}}+\sqrt{L_{2}}\right) \pm j \omega\left(\sqrt{L_{3}} \mp \sqrt{L_{2}}\right)}{-j \omega\left(\sqrt{L_{3}} \mp \sqrt{L_{2}}\right)^{2}-R_{S C}}\right\} I_{1}
\end{aligned}
$$

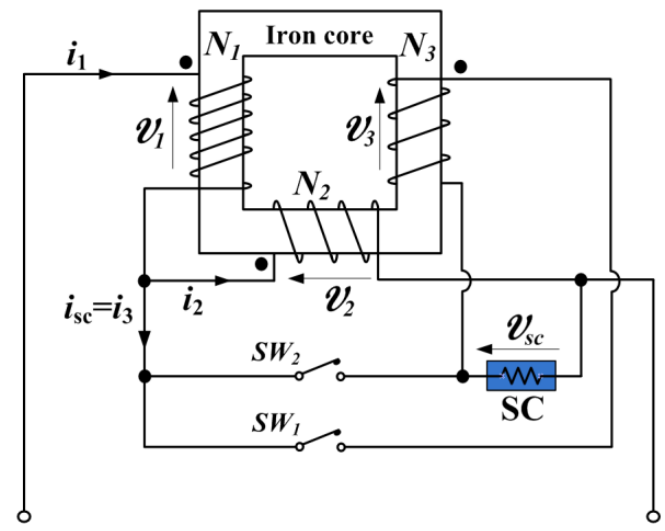

Fig. 1. Structure of the series-connection-type SFCL between two coils with an additional third winding.

$$
\begin{aligned}
= & \frac{j \omega\left(\sqrt{L_{1}}+\sqrt{L_{2}}\right)^{2}\left\{-j \omega\left(\sqrt{L_{3}} \mp \sqrt{L_{2}}\right)^{2}-R_{S C}\right\}}{-j \omega\left(\sqrt{L_{3}} \mp \sqrt{L_{2}}\right)^{2}-R_{S C}} I_{1} \\
& +\frac{( \pm j \omega)^{2}\left(\sqrt{L_{1}}+\sqrt{L_{2}}\right)^{2}\left(\sqrt{L_{3}} \mp \sqrt{L_{2}}\right)^{2}}{-j \omega\left(\sqrt{L_{3}} \mp \sqrt{L_{2}}\right)^{2}-R_{S C}} I_{1} \\
Z_{S F C L}= & \frac{V_{S F C L}}{I_{1}}=\frac{j \omega R_{S C}\left(\sqrt{L_{1}}+\sqrt{L_{2}}\right)^{2}}{R_{S C}+j \omega\left(\mp \sqrt{L_{2}}+\sqrt{L_{3}}\right)^{2}}
\end{aligned}
$$

Here, $\omega$ is the angular frequency. The magnitude of the fault current with the current limiting operation of the proposed SFCL can derived from the electrical equivalent model from Fig. 2. It can be set by adjusting the critical current value $\left(I_{c}\right)$ of the superconducting elements that are series connection with the primary and secondary wiring inductance $\left(L_{1}, L_{2}\right)$, the secondary and tertiary wiring inductance $\left(L_{2}, L_{3}\right)$ and the tertiary wiring.

$$
\begin{aligned}
& I_{2}= \frac{j \omega M_{12} \mp j \omega M_{13}}{ \pm j \omega M_{23}-j \omega L_{2}} I_{1}+\frac{ \pm j \omega M_{23}-j \omega L_{3}-R_{s c}}{ \pm j \omega M_{23}-j \omega L_{2}} I_{3} \\
& I_{1}= I_{2}+I_{3} \\
&= \frac{j \omega M_{12} \mp j \omega M_{13}}{ \pm j \omega M_{23}-j \omega L_{2}} I_{1} \\
&+\frac{ \pm j \omega M_{23}-j \omega L_{3}-R_{s c} \pm j \omega M_{23}-j \omega L_{2}}{ \pm j \omega M_{23}-j \omega L_{2}} I_{3} \\
&\left(\sqrt{L_{1}}+\sqrt{L_{2}}\right)\left( \pm j \omega \sqrt{L_{3}}-j \omega \sqrt{L_{2}}\right) I_{1} \\
&=\left\{\left(\mp \sqrt{L_{3}}+\sqrt{L_{2}}\right)\left( \pm j \omega \sqrt{L_{3}}-j \omega \sqrt{L_{2}}\right)-R_{s c}\right\} I_{3} \\
&\left(\sqrt{L_{1}}+\sqrt{L_{2}} I_{1}=\left(\mp \sqrt{L_{3}}+\sqrt{L_{2}}\right) I_{3}\right. \\
& I_{1}=\frac{\sqrt{L_{2}} \mp \sqrt{L_{3}}}{\sqrt{L_{1}}+\sqrt{L_{2}}} I_{C}
\end{aligned}
$$

Here, "+" represents the case of the subtractive winding between the secondary and third coils, while "-" represents the case of the additive winding between the secondary and third coils. Note that the winding between the primary and secondary coils is a case of additive polarity winding. One of the important factors for the analysis of the current limiting and recovery characteristics with additive polarity is that it is additionally connected to the series-connection-

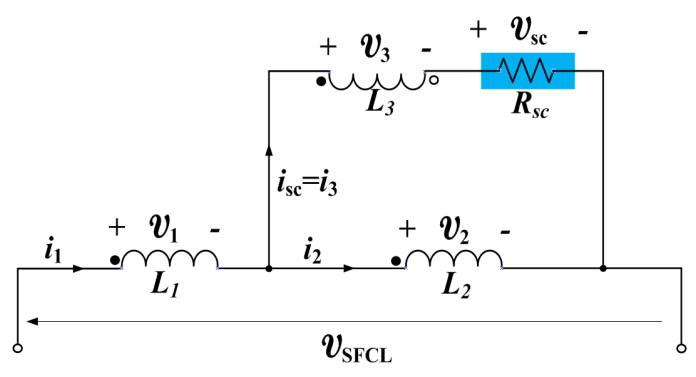

Fig. 2. Electrical equivalent circuit of the series-connectiontype SFCL between the two coils with an additional third winding. 
type SFCL structure, which has the conventional magnetic coupling.

\section{Experimental Results and Discussion}

\subsection{Experimental preparation}

To analyze the current limiting and recovery characteristics of the proposed SFCL, we considered a shortcircuit simulation circuit that had a power supply $E_{I n}$ of 60 $\mathrm{Hz}$, a line impedance of $Z_{I n}$, and a load impedance of $Z_{L}$ as shown in Fig. 3. To simulate a fault at the load terminal, $E_{I n}$ was applied by insertion of the switch $S_{l}$, and, after inserting of $S_{l}$, a short circuit was simulated by insertion of switch $S_{2}$. In addition, the fault was eliminated by opening after insertion of $S_{2}$ for 5 periods.

A $\mathrm{YBa}_{2} \mathrm{Cu}_{3} \mathrm{O}_{7-\delta}$ (YBCO) film that had the critical temperature of $87 \mathrm{~K}$ was used as the HTSC element with a parallel connection for the secondary coil and a series connection for the third coil. To protect the thin film device from the heat generated during quenching, a $200 \mathrm{~nm}$-wide $\mathrm{Au}$ layer mounted HTSC element is used, and, at that time, the critical current value $I_{C}$ was $27 \mathrm{~A}$.

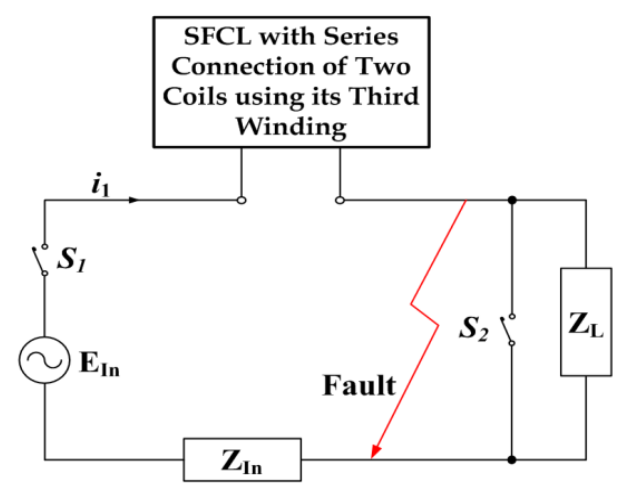

Fig. 3. Structure of the experimental circuit to simulate a fault generation.

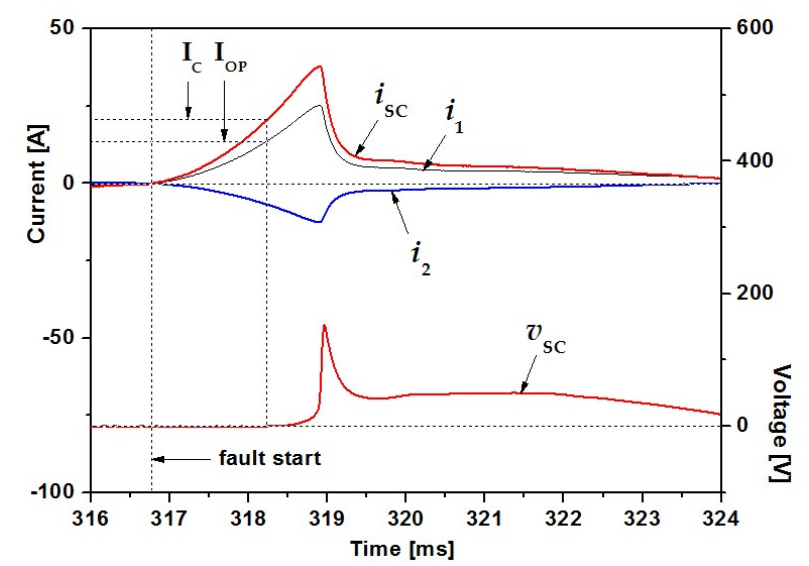

Fig. 4. Fault current limit characteristics of the seriesconnection-type SFCL without third winding.

\subsection{Results and discussion}

Fig. 4 shows the expanded current waveforms of the HTSC element and each of the coils for the SFCL that had magnetic coupling with an additive winding between the primary and secondary coils without using the third coils immediately after fault generation. It can be observed that the current flowing through the secondary coil was the reverse of the current flowing through the primary coil due to the additive winding between the primary and secondary coils. Immediately after fault generation, the current flowing through the HTSC element steadily increased. The voltage was induced by the generation of the quench to the HTSC element above the critical current value, and the fault current was limited by generation of the operation current $I_{O P}$.

Fig. 5 shows the current limiting and recovery characteristics of the series-connection-type SFCL that has magnetic coupling in which the primary and secondary coils have additive polarity winding without an additional third coil. Although Fig. 5(a) shows that the voltages
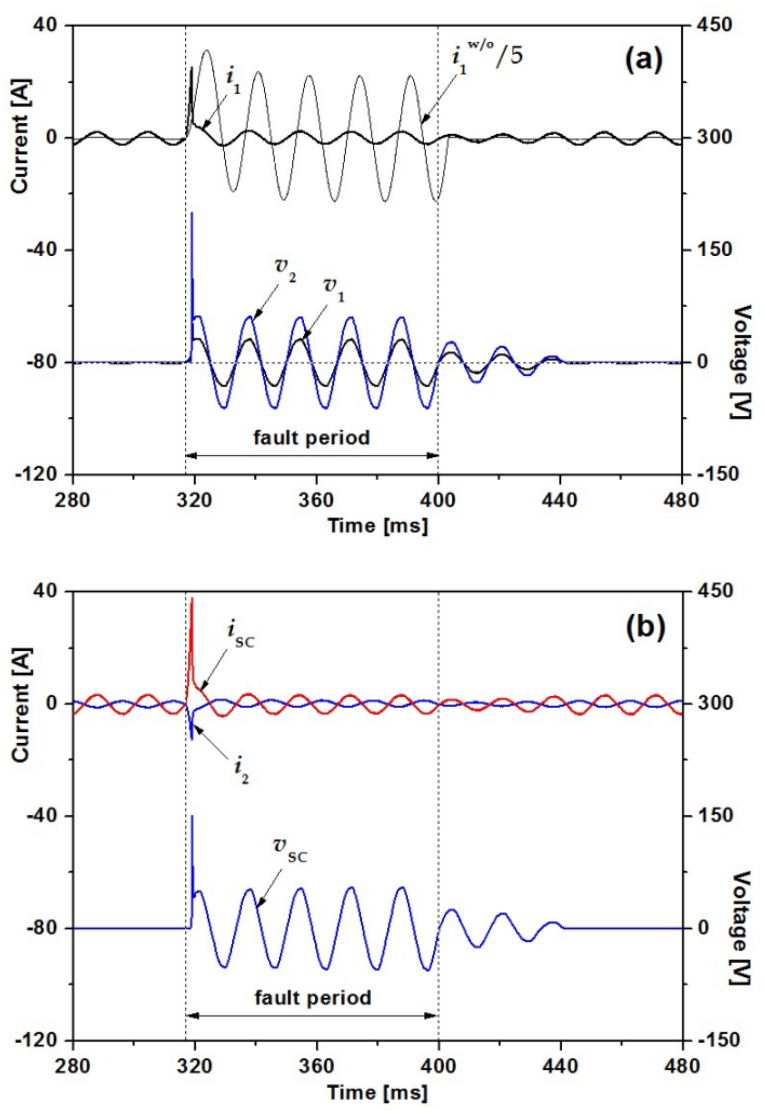

Fig. 5. Current limiting and recovery characteristics of the series connection SFCL without the third winding: (a) Induced voltage and limited conducting current; (b) Waveform of the induced voltage to the HTSC element, and waveforms of the current flowing through the secondary coil and the current flowing through the HTSC element. 
induced to coils 1 and 2 are in phase, the voltage magnitude of coil 1 is smaller than the voltage induced to coil 2. Also, the fault current limiting performance is better that when there is no SFCL during the fault generation period. Fig. 5(b) shows that the voltage induced to the HTSC element is identical the voltage induced to coil 2. Even though the current flowing through the HTSC element is in phase with the current flowing through coil 1, the current flowing through the HTSC element is reverse to the current flowing through coil 2 because coils 1 and 2 are cases of additive polarity winding. In addition, it takes about 2.5 periods for the HTSC element to recover to the superconducting state after the fault is eliminated.

Fig. 6 shows the fault current limiting characteristics when the additional coil 3 is artificially connected in parallel to coil 3 in the structure of the series-connectiontype SFCL with additive polarity winding between coils 1 and 2. It shows that the voltage magnitude induced to the HTSC element for the additive polarity winding case is smaller than that for the subtractive polarity winding case. In other words, the burden of the HTSC element is larger for the subtractive polarity winding case. As seen in Fig. $6(\mathrm{a})$, when there is additive polarity winding between coils 2 and 3 , the current flowing through coil 2 is reverse
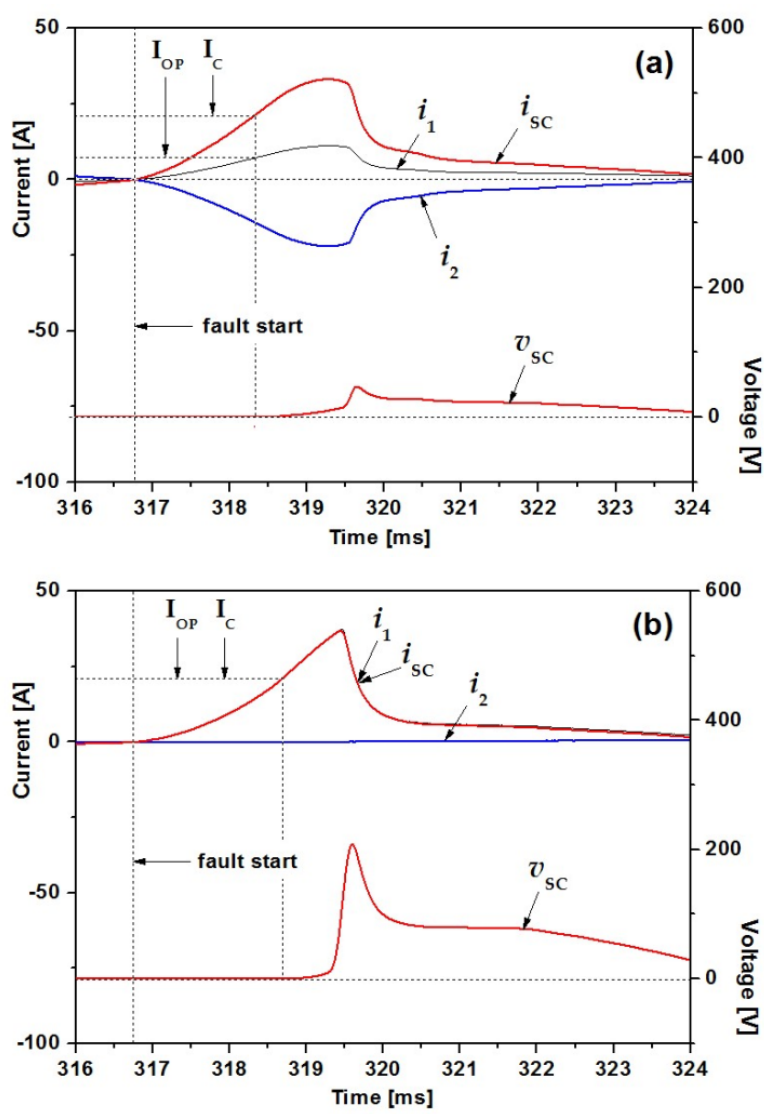

Fig. 6. Fault current limiting characteristics of the series connection SFCL with the third winding: (a) The additive polarity winding case; (b) The subtractive polarity winding case. to the current flowing through coil 3 , and the negative current magnitude flowing through $i_{2}$ is two times larger than $i_{2}$ of the SFCL structure without the third winding. However, in the case of the subtractive polarity winding, as demonstrated in Fig. 6(b), the current flowing through coil 2 becomes close to 0 , and the current of coil 1 is identical to the current flowing through the HTSC element. This happens because the negative current flowing through coil 2 and the positive current flowing through coil 3 mutually cancel each other out. Finally, the current flowing through coil 2 becomes zero. As a result, since all of the current flowing through coil 1 flow into coil 3 and not into coil 2, $i_{2}$ and $i_{S C}$ becomes identical.

Fig. 7 shows the current limiting and recovery characteristics of the series connection SFCL with magnetic coupling and additive polarity winding between coils 2 and 3 and an additional third coil. Even though Fig. 7(a) shows that the voltages induced to coils 1 and 2 are in phase, the induced voltage of coil 1 is smaller than that of coil 2. Also,
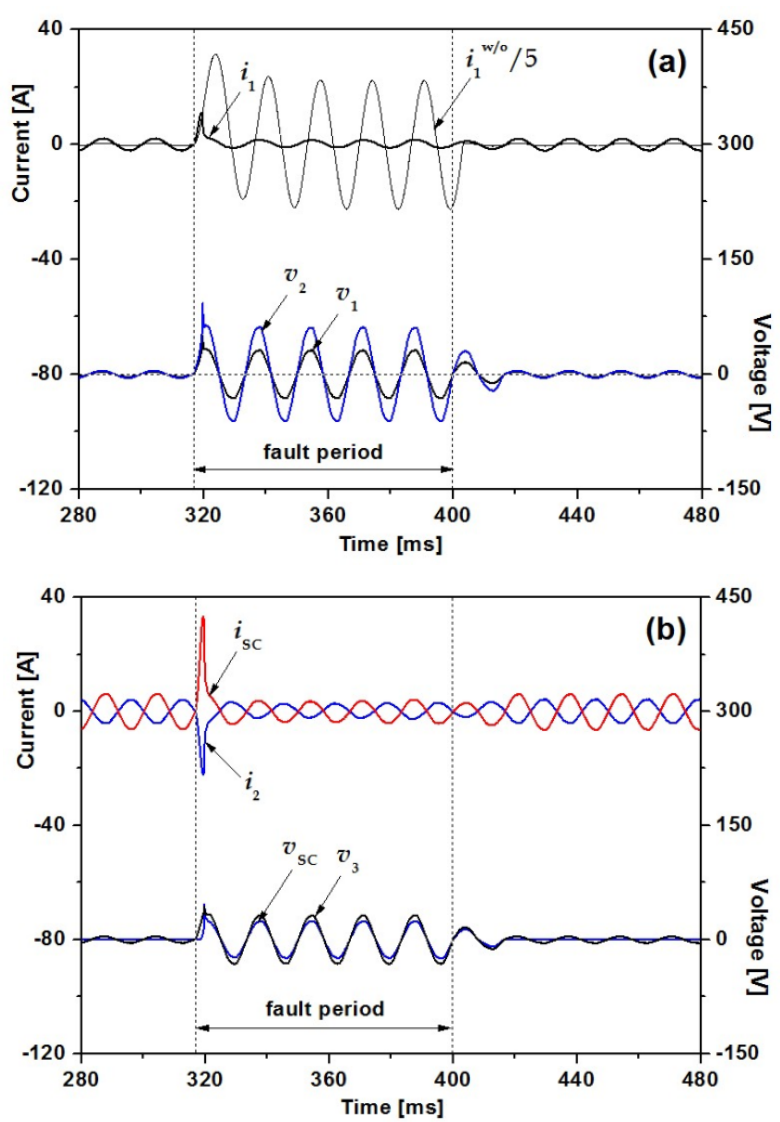

Fig. 7. Current limiting and recovery characteristics with additive polarity winding between coils 2 and 3 when the third winding was added: (a) Induced voltages to the primary and secondary coils and the limited conducting current; (b) Waveforms of the induced voltage to the HTSC element and the third coil, and waveforms of the current flowing through the secondary coil and the current of the HTSC element. 
the fault current limiting performance is better than that achieved without the SFCL during the fault generation period.

Fig. 7(b) shows that the induced voltage of the HTSC element is slightly smaller than the induced voltage of coil 3. Even though the current flowing through the HTSC element is in phase with the current flowing through coil 1 , it is inverse of the current flowing through coil 2, and the magnitude becomes larger because the negative currents are added together due the additive polarity winding of coils 2 and 3. Also, it can be seen that it takes about one period of time for the HTSC element to recover to the superconducting state after fault elimination. The measured results show that the recovery and fault current limiting characteristics improved with the additive polarity winding between coils 2 and 3 when the third coil was added in comparison to the case without the third coil.

However, as verified in Fig. 8, when the third coil was added and there was subtractive polarity winding between
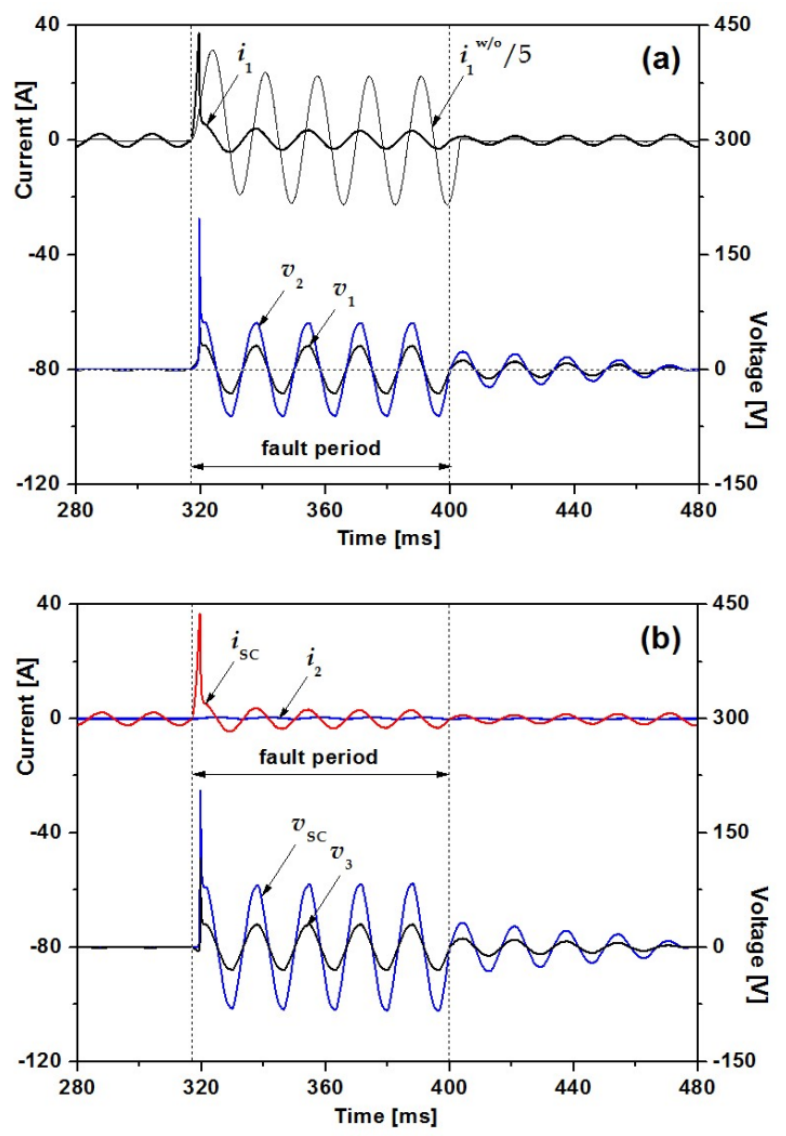

Fig. 8. Current limiting and recovery characteristics with subtractive polarity winding between coils 2 and 3 when the third winding was added: (a) Induced voltages to the primary and secondary coils and the limited conducting current; (b) Waveforms of the induced voltage to the HTSC element and the third coil, and waveforms of the current flowing through the secondary coil and the current of the HTSC element.

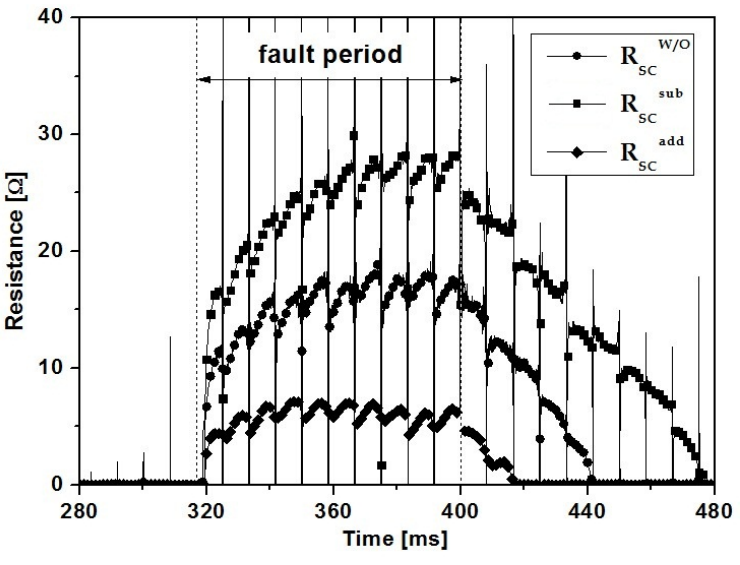

Fig. 9. Resistance curves of the HTSC element according to the winding direction of the series-connectiontype SFCL with the third winding.

coils 2 and 3, the recovery characteristics of the HTSC element and the fault current limiting characteristics were not improved. This may be due to the fact the negative current flowing through coil 2 and the positive current through coil 3 cancel each other out, which finally makes the current flowing through coil 2 become zero.

Fig. 9 shows the resistance generation curves according to the fault generation recovery of the HTSC element in relation to the winding direction between the secondary and third coils. For comparison, the resistance generation curve of the HTSC element in relation to the secondary and third windings when the third winding was added and the resistance generation curve of the HTSC element without the third winding are shown together. It can be seen that there was faster recovery for the additive polarity winding case between the secondary and third winding than for the subtractive polarity winding case. The analysis above verified that the recovery characteristics improved because there was faster recovery in the case of the additive polarity winding with the third winding than without the third winding. This work clearly demonstrated that additive polarity winding allows better SFCL performance in terms of the current limiting aspects and the recovery aspects in comparison to a structure with subtractive polarity winding.

\section{Conclusion}

This paper analyzed the recovery and current limiting characteristics of a flux-lock type SFCL with magnetic coupling with a series connection between coils 1 and 2 . Our analysis showed that the current limiting and recovery performance of the SFCL was greatly improved with additive polarity winding between coils 2 and 3 when a third winding was added in comparison to the case with subtractive polarity winding. However, in the case of the subtractive polarity winding, it was verified that the fault current limiting and the recovery performance of the HTSC 
element was not improved in comparison to the case without the third winding. This may be due to the fact that the current flowing through the secondary winding becomes zero because $L_{1}$ and $L_{3}$ have the series connection structure of the subtractive polarity winding case.

\section{Acknowledgements}

This work was supported by the Human Resources Development program (No. 20154030200940) of the Korea Institute of Energy Technology Evaluation and Planning (KETEP) grant funded by the Korea Government Ministry of Trade, Industry and Energy.

\section{References}

[1] S. H. Lim, H. S. Choi, D. C. Chung, Y. H. Jeong, Y. H. Han, T. H. Sung, and B. S. Han, "Fault current limiting characteristics of resistive type SFCL using a transformer," IEEE Trans. Appl. Supercond., vol. 15, no. 2, pp. 2055-2058, June 2002.

[2] H. Kado, M. Ichikawa, M. Shibuya, M. Kojima, M. Kawahara, and T. Matsumura, "Inductive type fault current limiter using Bi-2223 thick film on $\mathrm{MgO}$ cylinder with Bi-2212 buffer Layer," IEEE Trans. Appl. Supercond., vol. 15, no. 2, pp. 2051-2054, June 2005.

[3] H. Shimizu, Y. Yokomizu, T. Matsumura, and N. Murayama, "Proposal of flux flow resistance type fault current limiter using Bi2223 high Tc superconducting bulk," IEEE Trans. Appl. Supercond., vol. 12, no. 1, pp. 876-879, March 2002.

[4] S. H. Lim, T. H. Han, S. W. Yim, H. S. Choi, and B.S. Han, "Current limiting characteristics of a fluxlock type SFCL dependent on fault angles and core saturation," IEEE Trans. Appl. Supercond., vol. 17, no. 2, pp. 1827-1830, June 2007.

[5] H. S. Choi and S. H. Lim, "Operating performance of the flux-lock and the transformer type superconducting fault current limiter using the YBCO thin films," IEEE Trans. Appl. Supercond., vol. 17, no. 2, pp. 1823-1826, June 2007.

[6] Seckcheol Ko, Sung-Hun Lim, and Tae-Hee Han, "Analysis on fault current limiting and recovery characteristics of a flux-lock type SFCL with an isolated transformer," Physica C, vol. 484, pp. 263266, January 2013.

[7] S. H. Lim and H. S. Choi, "Quench characteristics of HTSC elements in series-connected flux-lock type SFCLs through magnetic flux-linkage," Physica $C$, vol. 445-448, pp. 1073-1077, October 2006.

[8] S. H. Lim, "Operational characteristics of flux-lock type SFCL with series connection of two coils,"
IEEE Trans. Appl. Supercon., vol. 17, no. 2, pp. 1895 1898, June 2007.

[9] S. H. Lim, "Comparative study on current limiting characteristics of flux-lock type SFCL with series or parallel connection of two coils," Physica C, vol. 468, pp. 2076-2080, September 2008.

[10] S. H. Lim, I. K. You, and J. C. Kim, "Study on peak current limiting characteristics of a flux-lock type SFCL using its third winding," IEEE Trans. Appl. Supercon., vol. 21, no. 3, pp. 1275-1279, June 2011.

[11] S. H. Lim, "Analysis on current limiting characteristics of a flux-lock type SFCL with two triggering current levels," Physica C, vol. 471, pp. 1354-1357, November 2011.

[12] S. H. Lim, S. C. Ko, and T. H. Han, "Analysis on current limiting characteristics of a transformer type SFCL with two triggering current levels," Physica $C$, vol. 484, pp. 253-257, January 2013.

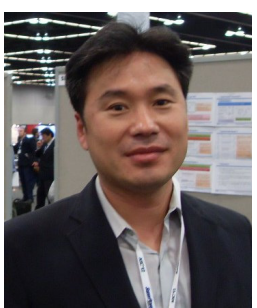

Seok-Cheol Ko He received his B.S., M.S., and Ph.D. degrees from Chonbuk National Univ., Korea in 1996, 2002, and 2005, respectively. Currently, he is a professor in the Industry-University Cooperation Foundation at Kongju National Univ., Korea.

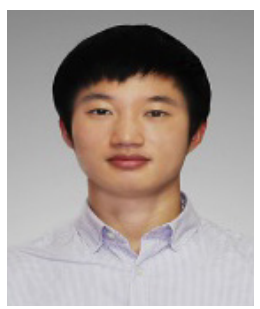

Young-Pil Kim He received his B.S. and M.S. degrees from Kongju National Univ., Korea in 2014 and 2016, respectively. Currently, he is doing a Ph. D. in the Div. of Electrical, Electronic and Control Engineering at Kongju National Univ., Korea.

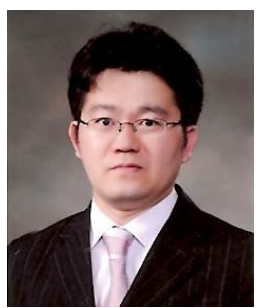

Sung-Hun Lim He received his B.S., M.S., and Ph.D. degrees from Chonbuk National Univ., Korea in 1996, 1998, and 2003, respectively. Currently, he is a professor in the Dept. of Electrical Engineering at Soongsil Univ., Korea. 